\title{
Assessment of the Relationship Between Serum Prostate- Specific Antigen Level and Serum Fasting Glucose, Total Cholesterol and Neutrophil-Lymphocyte Ratio in Men Aged 50-70 Years with Prostate-Specific Antigen Level 0-10 ng/mL without Prostate Cancer Diagnosis
}

\author{
(D) Bora Irer MD \\ Izmir Metropolitan Municipality Eșrefpașa Hospital, Clinic of Urology, Izmir, Turkey
}

\begin{abstract}
Objective: To evaluate the relationship between serum prostate-specific antigen (PSA) level and total cholesterol, fasting blood glucose, and neutrophillymphocyte ratio (NLR) in men without prostate cancer.

Materials and Methods: Between 2010 and 2017, 2631 male participants aged 50-70 years with a serum PSA level of 0-10 ng/mL were included from a population of 4643 healthy males who participated in a health screening program conducted by the Izmir Metropolitan Municipality Eşrefpaşa Hospital in the district villages of Izmir. Participants' serum PSA, fasting blood glucose, total cholesterol levels, and NLR were retrospectively assessed. Participants were grouped as those with high and low serum PSA levels, high and low glucose levels, and high and low cholesterol levels. Differences between the groups in terms of serum PSA levels, serum total cholesterol, glucose, and NLR were analyzed.

Results: The mean age of the participants was $60.2 \pm 5.4$ years and the mean serum PSA level was $1.28 \pm 1.20 \mathrm{ng} / \mathrm{mL}$. The mean PSA value was higher in the high cholesterol group $(1.36 \pm 1.33$ vs $1.19 \pm 1.02, \mathrm{p}<0.001)$ compared to the low cholesterol group, and the mean PSA value in the high glucose group was lower than in the low glucose group $(1.08 \pm 0.86$ vs $1.32 \pm 1.25, \mathrm{p}<0.001)$. Compared to the normal PSA group, the high PSA group had higher mean cholesterol level (208.6 \pm 39.9 vs $203.3 \pm 41.8, p<0.001)$ and NLR $(2.17 \pm 1.00$ vs $2.06 \pm 0.89, p=0.039)$, but lower glucose level $(108.5 \pm 32.5$ vs 117.2 $\pm 51.0, p=0.004)$. Serum PSA level was positively correlated with total cholesterol and $N L R(r=0.074$ and $p<0.001, r=0.050$ and $p=0.011)$, and negatively with glucose level $(r=-0.084$ and $p<0.001)$.

Conclusion: Evaluation of total cholesterol, fasting glucose, and NLR, which may be associated with serum PSA levels, may help urologists when investigating elevated serum PSA levels in asymptomatic men aged 50-70 years who have not been diagnosed with prostate cancer.
\end{abstract}

Keywords: Prostate-specific antigen testing, prostate cancer, screening

\section{Introduction}

Globally, prostate cancer is the second leading cause of cancerrelated mortality in males $(1,2)$. The incidence of prostate cancer has risen in many countries over the past two decades due to prostate cancer screening utilizing the serum prostate-specific antigen (PSA) test $(3,4,5)$. Although PSA testing is not currently recommended as a routine screening tool for prostate cancer 
$(6,7,8)$, it is commonly used as a preliminary test for men who present to urology outpatient clinics with complaints regarding urination or in patients over 40 years of age upon their request. The PSA glycoprotein, which is secreted from the prostate gland, has no definitive threshold value for prostate cancer, and high serum PSA levels can occur in benign prostatic hypertrophy $(\mathrm{BPH})$, prostatitis, and following procedures performed on the prostate (prostate massage, prostate biopsy, and urethroscopy) (9).

Serum PSA levels may be affected by a number of factors other than prostate disorders in men who have not been diagnosed with prostate cancer. Although there are studies in the literature investigating the effects of obesity, serum glucose, total cholesterol, and triglyceride levels on serum PSA levels, the results of these studies are contradictory $(10,11,12,13)$. Today, many men aged 50-70, despite not being diagnosed with diabetes or hypercholesterolemia, are found to have high blood glucose or total cholesterol level when tested for PSA.

In addition to numerous other parameters, neutrophillymphocyte ratio (NLR) has recently been studied as a marker of the inflammatory response in solid organ tumors because it is easily determined. NLR is an established marker of progression and poor prognosis in lung, pancreatic, colorectal, ovarian, and prostate cancer (14). Although there are many studies demonstrating a relationship between NLR and the risk of prostate cancer diagnosis, recurrence, and progression $(15,16)$, few studies have shown a relationship between serum PSA levels and NLR in patients without prostate cancer (14).

The aim of this study was to determine the relationship between serum PSA level and fasting blood glucose, total cholesterol, and NLR in men aged 50-70 years without prostate cancer.

\section{Materials and Methods}

Of 4643 healthy males who presented for health screening conducted by the İzmir Metropolitan Municipality Eşrefpaşa Hospital in the district villages of Izmir between 2010 and 2017, 2631 participants aged 50-70 with a serum PSA level of 0-10 $\mathrm{ng} / \mathrm{mL}$ were included in the study. The participants' serum PSA, fasting blood glucose, total cholesterol levels, and NLR were retrospectively evaluated. Patients with prostate cancer and patients receiving medical treatment for urinary tract infections, $\mathrm{BPH}$, diabetes, and hyperlipidemia were excluded from the study. Relationships between serum PSA level and fasting glucose, total cholesterol, and NLR were assessed. Participants were grouped as those with and without high glucose $(>126$ $\mathrm{mg} / \mathrm{dL}$ ) (17) and high cholesterol (>200 mg/dL) (18), and the differences between groups and their association with serum PSA levels were assessed. In addition, differences in serum total cholesterol, glucose, and NLR between participants with and without serum PSA level over $2.5 \mathrm{ng} / \mathrm{mL}$ (8) were evaluated.

Because the study was designed as a retrospective chart review, ethics committee approval and informed consent were not obtained. However, the participants provided informed consent during the health screening.

\section{Results}

The mean age of the 2631 male participants included in the study was $60.2 \pm 5.4$ years and their mean serum PSA level was $1.28 \pm 1.20 \mathrm{ng} / \mathrm{mL}$. The participants' demographic data, serum glucose and total cholesterol levels, and NLR are shown in Table 1. Men with high cholesterol had significantly higher mean serum PSA levels than men without high cholesterol $(p<0.001)$. However, mean serum PSA level was significantly lower in participants with high serum glucose levels when compared to those without high serum glucose $(p<0.001)$ (Table 2). Comparison of groups with and without elevated PSA revealed higher total cholesterol and NLR and lower mean fasting glucose levels among men with elevated serum PSA levels ( $p<0.001, p=0.039$, and $p=0.004$, respectively) (Table 3 ). There were statistically significant but weak positive correlations between serum PSA level and total cholesterol and NLR $(r=0.074$ and $p<0.001 ; r=0.050$ and $p=0.011)$. However, there was a significant but weak negative correlation between serum PSA level and fasting blood glucose $(r=-0.084$ and $p<0.001)$.

\begin{tabular}{|l|l|}
\hline \multicolumn{2}{|l|}{ Table 1. Demographic data of the participants } \\
\hline Number of participants & 2631 \\
\hline Mean age (range), years & $60.2 \pm 5.4(50-69)$ \\
\hline Mean PSA (range), $\mathbf{n g} / \mathbf{m L}$ & $1.28 \pm 1.20(0.10-9.32)$ \\
\hline Mean cholesterol (range), $\mathbf{~ g / d L ~}$ & $203.9 \pm 41.6(83-437)$ \\
\hline Mean glucose (range), $\mathbf{~ g / d L ~}$ & $116.2 \pm 49.3(46-554)$ \\
\hline Mean neutrophil/lymphocyte ratio & $2.07 \pm 0.91$ \\
\hline $\begin{array}{l}\text { Elevated glucose (>126 } \mathbf{~ m g / d L ) , ~ ( n , ~ \% ) ~} \\
\text { Yes }\end{array}$ & $2153,81.8 \%$ \\
\hline $\begin{array}{l}\text { Elevated cholesterol }(>\mathbf{2 0 0} \mathbf{~ m g / d L ) , ~ ( n , ~ \% ) ~} \\
\text { No } \\
\text { Yes }\end{array}$ & $237,11.3 \%$ \\
\hline PSA: Prostate-specific antigen & $1263,48.0 \%$ \\
\hline
\end{tabular}

\begin{tabular}{|c|c|c|c|c|c|}
\hline \multicolumn{3}{|c|}{ Elevated glucose } & \multicolumn{3}{|c|}{ Elevated cholesterol } \\
\hline $\begin{array}{l}\text { Yes } \\
n=237\end{array}$ & $\begin{array}{l}\text { No } \\
n=2153\end{array}$ & $p$ value & $\begin{array}{l}\text { Yes } \\
n=1368\end{array}$ & \begin{tabular}{|l} 
No \\
$n=1263$
\end{tabular} & $p$ value \\
\hline $61.2 \pm 5.1$ & $60.0 \pm 5.5$ & $<0.001$ & $60.3 \pm 5.5$ & $60.2 \pm 5.4$ & 0.236 \\
\hline $1.08 \pm 0.86$ & $1.32 \pm 1.25$ & $<0.001$ & $1.36 \pm 1.33$ & $1.19 \pm 1.02$ & $<0.001$ \\
\hline
\end{tabular}


Bora İrer

Serum and Blood Parameters Affecting Serum Prostate-Specific Antigen Level

\begin{tabular}{|c|c|c|c|}
\hline & $\begin{array}{l}\mathrm{PSA}>2.5 \\
\mathrm{ng} / \mathrm{mL} \\
\mathrm{n}=297\end{array}$ & $\begin{array}{l}\mathrm{PSA}<2.5 \\
\mathrm{ng} / \mathrm{mL} \\
\mathrm{n}=2334\end{array}$ & $p$ value \\
\hline Age & $63.1 \pm 4.6$ & $59.9 \pm 5.4$ & $<0.001$ \\
\hline PSA & $4.01 \pm 1.46$ & $0.93 \pm 0.52$ & $<0.001$ \\
\hline Cholesterol & $208.6 \pm 39.9$ & $203.3 \pm 41.8$ & $<0.001$ \\
\hline Glucose & $108.5 \pm 32.5$ & $117.2 \pm 51.0$ & 0.004 \\
\hline Neutrophil/lymphocyte ratio & $2.17 \pm 1.00$ & $2.06 \pm 0.89$ & 0.039 \\
\hline
\end{tabular}

\section{Discussion}

PSA is a glycoprotein tumor marker that is now commonly used in prostate cancer diagnosis and follow-up. Although it is secreted from normal prostate cells, prostate cancer cells secrete 10 times more PSA than normal (19). In addition to prostate cancer, PSA levels have also been observed in benign prostate diseases such as $\mathrm{BPH}$ and prostatitis, and interventions involving the prostate such as prostate biopsy, prostate resection, rigid cystoscopy, and transrectal ultrasonography (19). Although not currently recommended for routine prostate cancer screening $(6,7,8)$, it is widely used in urology outpatient clinics for those over 40 years of age upon patient request. Tawfik (20) argued that PSA testing creates high costs for the health care system, both due to the test itself and expenses associated with staging and treatment of detected prostate cancers. Based on these concerns, various studies were designed to determine when and for whom PSA testing should be conducted, which PSA value should be used as an indication for biopsy, and what factors affect serum PSA levels, but no definitive conclusions have been reached.

Despite numerous studies investigating associations between prostate cancer and hypercholesterolemia and statin use $(21,22,23)$, there have been relatively few studies aiming to correlate hypercholesterolemia with serum PSA levels in men without prostate cancer (12). Similar to the results of our study, Mondul et al. (12) investigated the relationship between serum total cholesterol and serum PSA levels in 2574 men over 40 years of age who did not have prostate cancer, and found that low PSA levels corresponded to low cholesterol levels. In addition, patients who achieved low cholesterol levels using statins also had low serum PSA levels. Higher cholesterol content and reduced cholesterol bioavailability in prostate tissue have been shown to alter the structure of cell membrane signaling sites and increase apoptosis in prostate cells $(24,25)$. Furthermore, these changes may also increase the inflammatory response against prostate cells. Therefore, both prostate cell apoptosis and the resulting inflammation can increase PSA release from prostate cells. Cholesterol is an important precursor for androgen synthesis (26). Androgens are shown to have a role in the proliferation of prostate cells and the development of prostate cancer, and PSA secretion has been associated with androgens (26). The cholesterol-androgen relationship may explain the association between elevated serum PSA and high cholesterol.
In this study, we showed that serum PSA levels were inversely correlated with fasting blood glucose and that glucose levels were lower in those with elevated PSA. Werny et al. (27) reported lower PSA levels in diabetic patients compared to non-diabetic patients. Müller et al. (10) showed that greater diabetes severity was associated with larger decreases in PSA. Similarly, Sun et al. (28) showed that patients with type 2 diabetes had lower PSA levels and that prediabetes was associated with higher PSA levels. Although the participants in the present study were not diagnosed with diabetes, the results were similar to those of previous studies. In addition, we found that participants with elevated PSA had lower fasting blood glucose levels. In the literature, molecular studies describing this inverse relationship between PSA and fasting blood glucose have shown a decline in blood testosterone level and increase in estrogen level in diabetes, which may result in a decrease in androgen-dependent PSA synthesis and secretion $(29,30)$.

Some previous studies have shown that systemic inflammation plays a role in the development and progression of cancer and that prostate cancer is associated with infectious agents, chronic infections, and dietary and hormonal inflammatory factors $(31,32)$. NLR is one of the markers of systemic inflammation and has been shown to correlate with the diagnosis, prognosis, and progression of prostate cancer $(15,16)$; however, the relationship between systemic inflammation and serum PSA levels in men without prostate cancer remains unclear. McDonald et al. (14) demonstrated in their study that NLR was associated with elevated serum PSA. In our study, there was a positive correlation between elevated serum PSA and NLR, and NLR was higher in the group with PSA elevation. NLR elevation is associated with higher concentrations of many pro-inflammatory cytokines. These pro-inflammatory cytokines cause cellular DNA damage which can lead to cell death or cancer development (32).

\section{Study Limitations}

The main limitations of this study are its retrospective nature, the lack of advanced testing to establish other possible etiologies of PSA elevation, and the lack of follow-up results for the participants.

\section{Conclusion}

The results of this study indicate that serum PSA elevation may be associated with high total cholesterol level, high NLR, and low fasting blood glucose level. Taking total cholesterol level, fasting glucose level, and NLR into account may help urologists when evaluating elevated serum PSA in asymptomatic men aged 50-70 who have never been diagnosed with prostate cancer. However, better planned studies that analyze the results of patient follow-up are needed to establish the exact relationship between these factors and serum PSA levels in asymptomatic men.

\section{Ethics}

Ethics Committee Approval: Because the study was designed as a retrospective chart review, ethics committee approval was not obtained. 
Informed Consent: Informed consent was obtained during the health screening in which the data analyzed in the study were collected. Separate informed consent was not sought for this retrospective chart review study.

Peer-review: Externally peer-reviewed.

Financial Disclosure: The author declared that this study received no financial support.

\section{References}

1. Ferlay J, Steliarova-Foucher E, Lortet-Tieulent J, et al. Cancer incidence and mortality patterns in Europe: estimates for 40 countries in 2012. Eur J Cancer 2013;49:1374-1403.

2. Jemal A, Siegel R, Xu J, Ward E. Cancer statistics, 2010. CA Cancer J Clin 2010;60:277-300.

3. Center MM, Jemal A, Lortet-Tieulent J, et al. International variation in prostate cancer incidence and mortality rates. Eur Urol 2012;61:1079-1092.

4. Bray F, Lortet-Tieulent J, Ferlay J, et al. Prostate cancer incidence and mortality trends in 37 European countries: an overview. Eur J Cancer 2010;46:3040-3052.

5. Etzioni R, Gulati R, Cooperberg MR, et al. Limitations of basing screening policies on screening trials: The US Preventive Services Task Force and Prostate Cancer Screening. Med Care 2013;51:295-300.

6. Hayes $\mathrm{JH}$, Barry MJ. Screening for prostate cancer with the prostate-specific antigen test: a review of current evidence. JAMA 2014;311:1143-1149.

7. Ilic D, Neuberger MM, Djulbegovic M, Dahm P. Screening for prostate cancer. Cochrane Database Syst Rev 2013:CD004720.

8. Mottet N, Bellmunt ], Bolla M, et al. EAU-ESTRO-SIOG Guidelines on Prostate Cancer. Part 1: Screening, Diagnosis, and Local Treatment with Curative Intent. Eur Urol 2017; 71:618-629.

9. Heindereih A, Bastian PJ, Bellmunt J, et al. EAU Guidelines on Prostate Cancer. Part 1: screening, diagnosis and local treatment with curative intent-update. Eur Urol 2014;65:124-137

10. Müller $H$, Raum E, Rothenbacher $D$, et al. Association of diabetes and body mass index with levels of prostate-specific antigen: implications for correction of prostate-specific antigen cutoff values? Cancer Epidemiol Biomarkers Prev 2009;18:1350-1356.

11. Naito M, Asai Y, Mori A, et al. Association of obesity and diabetes with serum prostate specific antigen levels in Japanese males. Nagoya J Med Sci 2012;74:285-292

12. Mondul AM, Selvin E, De Marzo AM, et al. Statin drugs, serum cholesterol, and prostate-specific antigen in the National Health and Nutrition Examination Survey 2001-2004. Cancer Causes Control 2010;21:671-678.

13. Oh JJ, Jeong SJ, Lee BK, et al. Does obesity affect the accuracy of prostate-specific antigen (PSA) for predicting prostate cancer among men undergoing prostate biopsy. BJU Int 2013;112:265-271 .

14. McDonald AC, Vira MA, Vidal AC, et al. Association between systemic inflammatory markers and serum prostate-specific antigen in men without prostatic disease - the 2001-2008 National Health and Nutrition Examination Survey. Prostate 2014;74:561-567.
15. Ceylan Y, Günlüsoy B, Degirmenci T, et al. Neutrophil-to-lymphocyte and neutrophil-to-monocyte rates in the decision for a prostate rebiopsy in patients with a previous benign pathology and consistently 2,5-10 ng/ml PSA value. Arch Esp Urol 2016;69:627-635.

16. Cao J, Zhu X, Zhao X, et al. Neutrophil-to-Lymphocyte Ratio Predicts PSA Response and Prognosis in Prostate Cancer: A Systematic Review and Meta-Analysis. PLoS One 2016;11:e0158770.

17. American Diabetes Association. 2. Classification and Diagnosis of Diabetes. Diabetes Care 2015:8-16.

18. Jellinger PS, Handelsman Y, Rosenblit PD, et al. American association of clinical endocrinologist and american collage of endocrinology guidelines for management of dyslipidemia and prevention of cardiovascular disease. Endocr Pract 2017;23(Suppl 2):1-87.

19. Duffy MJ. Prostate-specific antigen: does the current evidence support its use in prostate cancer screening? Ann Clin Biochem 2011;48:310-316.

20. Tawfik A. Prostate-Specific Antigen (PSA)-Based Population Screening for Prostate Cancer: An Economic Analysis. Ont Health Technol Assess Ser 2015;15:1-37

21. Schnoeller TJ, Jentzmik F, Schrader AJ, Steinestel J. Influence of serum cholesterol level and statin treatment on prostate cancer aggressiveness. Oncotarget 2017;8:47110-47120.

22. Farwell WR, D'Avolio LW, Scranton RE, et al. Statins and prostate cancer diagnosis and grade in a veterans population. J Natl Cancer Inst 2011;103:885-892.

23. Platz EA, Till C, Goodman PJ, et al. Men with low serum cholesterol have a lower risk of high-grade prostate cancer in the placebo arm of the prostate cancer prevention trial. Cancer Epidemiol Biomarkers Prev 2009; 18:2807-2813.

24. Hager $\mathrm{MH}$, Solomon KR, Freeman MR. The role of cholesterol in prostate cancer. Curr Opin Clin Nutr Metab Care 2006;9:379-385.

25. Schaffner CP. Prostatic cholesterol metabolism: regulation and alteration. Prog Clin Biol Res 1981;75:279-324.

26. Moon JY, Choi MH, Kim J. Metabolic profiling of cholesterol and sex steroid hormones to monitor urological diseases. Endocr Relat Cancer 2016;23:455-467.

27. Werny DM, Saraiya M, Gregg EW. Prostate-specific antigen values in diabetic and nondiabetic US men, 2001-2002. Am J Epidemiol 2006; 164:978-983.

28. Sun A, Liu R, Sun G. Serum prostate-specific antigen levels in men with prediabetes: a cross-sectional study. Scand J Clin Lab Invest 2015;75:273-281.

29. Djavan B, Waldert M, Seitz C, Marberger M. Insulin-like growth factors and prostate cancer. World J Urol 2001;19:225-233.

30. Betancourt-Albrecht $M$, Cunningham GR. Hypogonadism and diabetes. Int J Impot Res 2003;15(Suppl 4):14-20.

31. Coussens LM, Werb Z. Inflammation and cancer. Nature 2002;420:860-867.

32. Shafique K, Proctor MJ, McMillan DC, et al. Systemic inflammation and survival of patients with prostate cancer: Evidence from the Glasgow Inflammation Outcome Study. Prostate Cancer Prostatic Dis 2012;15:195-201. 